JLAB-THY-98-25

LA PLATA-TH 98/11

nucl-th/9806048

\title{
EQUATION OF STATE OF HADRONIC MATTER WITH DIBARYONS IN AN EFFECTIVE QUARK MODEL
}

\author{
Ricardo M. AGUIRRE 啁 and Martin SCHVELLINGER ${ }^{a, b, c}$ 舟 \\ a Physics Department, University of La Plata, C.C. 67, (1900) La Plata, Argentina. \\ ${ }^{b}$ The Nuclear/High Energy Physics Research Center, Hampton University, \\ Hampton, VA 23668, USA. \\ c Thomas Jefferson National Accelerator Facility, 12000 Jefferson Avenue, Newport \\ News, VA 23606, USA.
}

\begin{abstract}
The equation of state of symmetric nuclear matter with the inclusion of non-strange dibaryons is studied. We pay special attention to the existence of a dibaryon condensate at zero temperature. These calculations have been performed in an extended quark-meson coupling model with densitydependent parameters, which takes into account the finite size of nucleons and dibaryons. A first-order phase-transition to pure dibaryon matter has been found. The corresponding critical density is strongly dependent on the value of the dibaryon mass. The density behavior of the nucleon and dibaryon effective masses and confining volumes have also been discussed.
\end{abstract}

PACS: 21.65.+f; 12.39.Ba; 24.85.+p; 14.20Pt

Keywords: Nuclear matter; Bag model; Dibaryons; Equation of state; Bose condensate.

*Electronic address: aguirre@venus.fisica.unlp.edu.ar

${ }^{\dagger}$ On leave from University of La Plata.

Electronic addresses: schvell@cebaf.gov and martin@venus.fisica.unlp.edu.ar 
Nuclear matter properties have been modeled by several kind of local relativistic effective lagrangians which basically use point-like representations of nucleons and mesons as the relevant degrees of freedom. The differences are essentially coming from the nucleon-scalar meson interaction. It should be desirable to describe nuclear matter from a fundamental theory like QCD, but it is wellkown that in the low energy limit QCD becomes non-perturvative. This situation has motivated the development of several effective models of quark interaction, among them there is the MIT bag model [1]. This model predicts the existence of some multibaryons and strange exotics [2]. The early work of Jaffe [3] has concentrated the attention of theoretical studies [8, [4] and the further development of experimental research looking for signals of strange and non-strange dibaryons. In reference [3] it has been shown that in the scheme of the MIT bag model the gluon-exchange force should be responsible of the existence of a stable six-quarks bound state. This particle, the so-called $H$-particle, is a flavor singlet $J^{\pi}=0^{+}$dihyperon with a mass of $2150 \mathrm{MeV}$ and strangeness -2 . The problem has also been treated in the non-perturbative framework of the Skyrme model where dibaryons have been considered as axially symmetric skyrmions [5]. Non-strange as well as strange dibaryons and another multibaryons have been studied as multiskyrmions [6]. The experimental activity concerned to this search has been increased in the last years, recent experiments have been developed at TRIUMF and CELCIUS [7]. Non-strange dibaryons which have a small width have been described as promising candidates for experimental searches [8].

On the other hand, models based on the quantum field theory of hadrons including non-strange dibaryons as effective degrees of freedom have been extensively studied [9], obtaining very interesting effects on the binding energy per particle as well as on the equation of state (EOS) of the system. In these hadronic effective lagrangians nucleons and dibaryons are treated as point-like particles represented by two independent effective fields, both of them interact by the exchange of scalar and vector neutral mesons using two different sets of coupling constants.

The purpose of this work is to include finite size effects on the EOS, taking into account the quark structure of the particles. As we will show later, several features of our results are in agreement with those obtained in reference [9]. Therefore, this fact seems to support that the dibaryon condensate is essentially a model-independent issue. We have selected the so-called quark-meson coupling model (QMC) [10], which in some way satisfies the above mentioned requirements. In this scheme we can perform a simultaneous description of nucleons and dibaryons using only current quarks and mesons as effective degrees of freedom. The model was early developed by Guichon [10] and it has been extensively applied to calculate nuclear matter [11] as well as finite nuclei [12] properties with successful results. It has also been used to evaluate nucleon structure functions [13. The naturalness of the QMC model has been studied using the dimensional analysis [14. Recently the density dependence of the parameters of the MIT bag have been 
phenomenologically modeled [15], alternatively a relationship with observables evaluated in the quantum field theory of hadrons have been stablished [16, 17].

In this work we have developed an extension of the QMC model described in reference [16, by including dibaryons represented as spherical MIT bags confining six quarks. The extended QMC lagrangian density with quark fields $q_{\alpha}(x)$ coupled to scalar $\sigma(x)$ and vector $\omega_{\mu}(x)$ neutral mesons, is written as follows

$$
\begin{gathered}
\mathcal{L}_{Q M C}(x)=\mathcal{L}_{N}(x)+\mathcal{L}_{D}(x)+\mathcal{L}_{\text {Mesons }}^{0}(x), \\
\mathcal{L}_{N}(x)=\left(\mathcal{L}_{N}^{0}(x)-B_{1}\right) \Theta_{V N}-\frac{1}{2} \sum_{\alpha=1}^{3} \bar{q}_{\alpha}(x) q_{\alpha}(x) \Delta_{S N}, \\
\mathcal{L}_{D}(x)=\left(\mathcal{L}_{D}^{0}(x)-B_{2}\right) \Theta_{V D}-\frac{1}{2} \sum_{\alpha=1}^{6} \bar{q}_{\alpha}(x) q_{\alpha}(x) \Delta_{S D} .
\end{gathered}
$$

Here $\Theta_{V N}$ and $\Theta_{V D}$ are the radial non-overlapping step functions which schematically confine the quarks inside spherical bags for nucleons and dibaryons, respectively. $B_{1}$ and $B_{2}$ are the so-called MIT bag constants associated with these particles. Within the standard QMC treatment $B$ is a constant, however it can be considered as function of the baryonic density, $\rho_{B}$. The terms proportional to the surface delta functions $\Delta_{S N}$ and $\Delta_{S D}$ ensure a zero flux of quark current through the bag surface. In this lagrangian we have defined the following terms

$$
\begin{gathered}
\mathcal{L}_{N}^{0}(x)=\sum_{\alpha=1}^{3} \bar{q}_{\alpha}(x)\left(i \gamma^{\mu} \partial_{\mu}-m_{\alpha}+g_{\sigma} \sigma(x)-g_{\omega} \gamma^{\mu} \omega_{\mu}(x)\right) q_{\alpha}(x), \\
\mathcal{L}_{D}^{0}(x)=\sum_{\alpha=1}^{6} \bar{q}_{\alpha}(x)\left(i \gamma^{\mu} \partial_{\mu}-m_{\alpha}+g_{\sigma} \sigma(x)-g_{\omega} \gamma^{\mu} \omega_{\mu}(x)\right) q_{\alpha}(x), \\
\mathcal{L}_{\text {Mesons }}^{0}(x)=\frac{1}{2}\left[\partial^{\mu} \sigma(x) \partial_{\mu} \sigma(x)-m_{\sigma}^{2} \sigma^{2}(x)\right]-\frac{1}{4} F^{\mu \nu}(x) F_{\mu \nu}(x)+\frac{1}{2} m_{\omega}^{2} \omega^{\mu}(x) \omega_{\mu}(x) .
\end{gathered}
$$

Here $g_{\sigma}$ and $g_{\omega}$ are the quark-meson coupling constants associated with $\sigma(x)$ and $\omega_{\mu}(x)$, respectively. In order to get the minimal set of free parameters we have assumed that quarks do not distinguish baryon or dibaryon bags, i.e. the coupling constants are the same in both of the equations for baryons and dibaryons. In that follows we deal with $u$ and $d$ massless quarks, furthermore we introduce the index $\nu=1,2$ to label quantities related to nucleons and dibaryons, respectively.

The normalized quark wave function for the fundamental state in a spherical bag of radius $R_{\nu}$ is given by

$$
q_{\nu}(\vec{r}, t)=\mathcal{N}_{\nu} e^{-i \epsilon_{\nu} t / R_{\nu}} \times\left(\begin{array}{c}
j_{0}\left(y_{\nu} r / R_{\nu}\right) \\
i \beta_{\nu} \vec{\sigma} \cdot \hat{\mathrm{r}} j_{1}\left(y_{\nu} r / R_{\nu}\right)
\end{array}\right) \frac{\chi_{\nu}}{\sqrt{4 \pi}},
$$

where $r$ is the distance from the center of the bag, $\chi_{\nu}$ is the quark spinor and the normalization constant is

$$
\mathcal{N}_{\nu}=\frac{y_{\nu}}{\sqrt{2 R_{\nu}^{3} j_{0}^{2}\left(y_{\nu}\right)\left[\Omega_{\nu}\left(\Omega_{\nu}-1\right)+R_{\nu} m_{\alpha}^{*} / 2\right]}} .
$$

The parameter associated with the quark mass is $m_{\alpha}^{*}=m_{\alpha}-g_{\sigma} \bar{\sigma}$, and the energy eigenvalue is written as

$$
\epsilon_{\nu}=\Omega_{\nu}+g_{\omega} \nu \bar{\omega} R_{\nu}
$$


where $\Omega_{\nu}=\sqrt{y_{\nu}^{2}+\left(R_{\nu} m_{\alpha}^{*}\right)^{2}}$, and $\bar{\sigma}, \bar{\omega}$ are the mean values of meson fields calculated in the Mean Field Approximation. The $y_{\nu}$ variable is fixed by the boundary condition at the bag surface $j_{0}\left(y_{\nu}\right)=\beta_{\nu} j_{1}\left(y_{\nu}\right)$ as in reference [1] and $\beta_{\nu}=\sqrt{\left(\Omega_{\nu}-R_{\nu} m_{\alpha}^{*}\right) /\left(\Omega_{\nu}+R_{\nu} m_{\alpha}^{*}\right)}$. The mass associated with the bag is given by

$$
M_{\nu}=\frac{3 \nu \Omega_{\nu}-z_{0 \nu}}{R_{\nu}}+\frac{4}{3} \pi B_{\nu} R_{\nu}^{3}
$$

The $B_{\nu}$ are the bag constants already introduced in Eqs.(2) and (3), while $z_{0 \nu}$ takes into account the zero point energy of the bag.

The usual procedure in $\mathrm{QMC}$ is to fix the nucleon bag parameters at zero baryon density to reproduce the experimental nucleon mass $M_{1}=M_{N}=939 \mathrm{MeV}$, simultaneously it is required that the equilibrium condition $d M_{1}(\bar{\sigma}) / d R=0$ must be fulfilled. An analogous method should be applied to dibaryons, however the experimental value of in-vacuum dibaryon mass $M_{D}$ has not been definitely confirmed yet, and at the present, only theoretical estimates are availables. Therefore, we leave $M_{D}$ as a parameter of the model. Since the $B_{\nu}$ are related to vacuum properties we assume that $B_{1}=B_{2}=B$, at all densities. Although the paramaters $z_{0 \nu}$ could also have a density dependence, in a previous work [16] it was found that $z_{01}$ remains approximately constant at the baryon densities below four times the nuclear matter saturation density. Consecuently we have taken $z_{0 \nu}$ as constants fixed at zero density for each kind of bag. Under these assumptions one can immediately get a relation for masses and radii : $R_{2}=\left(M_{2} / M_{1}\right)^{1 / 3} R_{1}$.

In order to obtain the density dependence of $B$ we have stablished an explicit relationship between nuclear matter observables evaluated in the extended QMC model and in pure hadronic models, as it has been described in detail in [16]. In the present work we have selected the Zimanyi-Moszkowski model [18] to describe the hadronic sector (as in reference [16]). Considering that the effective nucleon mass predicted by the extended QMC model and the hadronic model must be the same, together with the fact that the outward quark-momentum on the bag surface must be compensated by the hadronic momentum going inside, one gets the following equations

$$
\begin{gathered}
M_{1}=M^{*}, \\
P_{b a g}=P_{h a d},
\end{gathered}
$$

which are valids at each value of baryon density. Here $M^{*}$ and $P_{h a d}$ are the effective nucleon mass and pressure of nuclear matter in the hadronic model, while $P_{b a g}=$ $-\left(d M_{1} / d R_{1}\right) / 4 \pi R_{1}^{2}$ is the pressure in the nucleon bag. Using these equations and $m_{\sigma}=$ $550 \mathrm{MeV}, m_{\omega}=783 \mathrm{MeV}, g_{\sigma}=4.576, g_{\omega}=2.222$ and $R_{1}^{0}=0.8 \mathrm{fm}$ we have obtained the density behaviour of $B=B\left(\rho_{B}\right)$ shown in Fig. 1 .

Once we have dinamically derived the parameter $B$ as a function of the baryon density, we can perform the calculations for nuclear matter with dibaryons. Firstly, we 
evaluate the energy per baryon of a system composed by symmetric nuclear matter with density $\rho_{N}$, and dibaryons with density $\rho_{D}$. Since each dibaryon carries baryon number two, the baryon density must be $\rho_{B}=\rho_{N}+2 \rho_{D}$. Using the quark wave functions of Eq.(7) one can construct the antisymmetrized (symmetrized) nucleon (dibaryon) physical states and evaluate the expectation value of the energy density $\mathcal{H}$ derived from the lagrangian of Eq.(1). For uniform matter the energy per baryon is given by

$$
\epsilon=\mathcal{H} / \rho_{B}=\frac{1}{\rho_{B}}\left[\frac{M_{1}^{4}}{\pi^{2}} F(\eta)+\frac{m_{\sigma}^{2} \bar{\sigma}^{2}}{2}+\frac{m_{\omega}^{2} \bar{\omega}^{2}}{2}+M_{2} \rho_{D}\right]
$$

where $\eta=k_{F} / M_{1}, k_{F}$ is the Fermi momentum for the nucleons and it is related to the nucleon density by $\rho_{N}=2 k_{F}^{3} / 3 \pi^{2}$, while $F(\eta)=\eta \sqrt{\eta^{2}+1}\left(2 \eta^{2}+1\right)-\log \left(\eta+\sqrt{\eta^{2}+1}\right)$. Chemical potentials for nucleons and dibaryons are given by $\mu_{N}=\sqrt{k_{F}^{2}+M_{1}^{2}}+3 g_{\omega} \bar{\omega}$ and $\mu_{D}=M_{2}+6 g_{\omega} \bar{\omega}$, respectively. Although the dibaryons are bosons, the corresponding particle number is conserved because they carry baryon charge and since in our lagrangian we have not included any decay mechanism. The pressure of hadronic matter at zero temperature can be written as

$$
P_{h a d}=\mu_{N} \rho_{N}+\mu_{D} \rho_{D}-\epsilon \rho_{B}
$$

The mean values of the meson fields have been evaluated by minimizing the energy, i.e. $\partial \epsilon / \partial \bar{\sigma}=0$ and $\partial \epsilon / \partial \bar{\omega}=0$, obtaining

$$
\bar{\sigma}=-\frac{1}{m_{s}^{2}}\left\{2 \frac{M_{1}^{2}}{\pi^{2}}\left[4 M_{1} F(\eta)-p_{F} \frac{d F}{d \eta}(\eta)\right] \frac{d M_{1}}{d \sigma}+\rho_{D} \frac{d M_{2}}{d \sigma}\right\},
$$

together with $\bar{\omega}=3 g_{\omega} \rho_{B} / m_{\omega}^{2}$.

In order to obtain numerical results we have arbitrarily selected the value of the in-vacuum dibaryon mass $M_{D}=1970 \mathrm{MeV}$. We have used the meson masses, free nucleon bag radius and the previously described procedure to calculate several in-vacuum quantities such as the parameters $z_{01}=3.27, z_{02}=6.27$, as well as the dibaryon bag radius $R_{2}^{(0)}=0.954 \mathrm{fm}^{-1}$, the usual boundary condition eigenvalue $y_{\nu}^{(0)}=2.042$ and the bag parameter $B^{(0)}=0.5546 \mathrm{fm}^{-4}$. When the constants $z_{01}, z_{02}$ have been fixed and the density dependent parameter $B$ has been obtained, we must find the effective bag radii $R_{1}\left(\rho_{B}\right)$ and $R_{2}\left(\rho_{B}\right)$. To do that we have impossed the equilibrium condition for bags immersed in hadronic medium in similar way as Eq.(12), but now using Eq.(14) in the right hand side. After a little algebra it gives

$$
4 \pi R_{\nu}^{4} P_{h a d}=-3 M_{\nu} R_{\nu}+4\left(3 \Omega_{\nu}-z_{0 \nu}\right)-3 a_{\nu} \frac{2 a_{\nu}\left(\Omega_{\nu}-1\right)+\Omega_{\nu}}{2 \Omega_{\nu}\left(\Omega_{\nu}-1\right)+a_{\nu}},
$$

where we have defined $a_{\nu}=m_{\alpha}^{*} R_{\nu}\left(\rho_{B}\right)$. It must be noticed that this condition becomes the standard equilibrium requirement of QMC model for vanishing $\rho_{B}$. 
Solving the boundary condition at the bag surface simultaneously with Eqs.(15) and (16), for a given baryon density and several values of the dibaryon abundance $Q=\rho_{D} / \rho_{B}$ we have evaluated the bag radii, masses, energy density and pressure as functions of $\rho_{B}$ and $Q$. The coupling constants have been fixed in order to reproduce at $Q=0$ the saturation density $\rho_{0}=0.15 \mathrm{fm}^{-3}$ and the binding energy per particle $\epsilon_{0}=16 \mathrm{MeV}$, obtaining $g_{\sigma}=4.6876$ and $g_{\omega}=2.2967$. In Fig. 1 the results for the nucleon bag radius $R_{1}$ are shown. From this we can see that at low densities the radius is an incresing function of $\rho_{B}$ and it becomes a smoothly decreasing function at densities around the saturation nuclear density. Furthermore, at densities below $1.4 \rho_{0}, R_{1}$ is higher than in-vacuum radius, notwithstanding for $Q=0$ the relative increment of $R_{1}$ remains under the value $2 \%$, as it is expected from $y$-scaling arguments in the analysis of quasi-elastic electron scattering [19. Therefore, the use of this radius and the nucleon effective mass $M_{1}$ is equivalent to have a bigger radius and the free nucleon mass, as it has been proposed in the reference of Sick [19]. Density variation of the radius is less pronounced as $Q$ increases. A similar behaviour for the dibaryon bag radius has been found.

To investigate the existence of a phase-transition from symmetric nuclear matter to a pure dibaryonic state we have used the Gibbs criterion. If there is a dynamical mechanism combining two nucleons to give one dibaryon, at the phase-transition point the conditions $2 \mu_{N}=\mu_{D}$ and $P_{n u c}=P_{d i b}$ must be fulfilled. Although we have not included such a reaction channel in our model, we are concerned with the stable initial and final phases rather than with any particular mechanism of dibaryon formation. Here $P_{n u c}$ and $P_{d i b}$ denote the pressure in pure nuclear and pure dibaryon matter, respectively. In Fig. 2 the quantities $2 \mu_{N}$ for $Q=0$ and $\mu_{D}$ for $Q=0.5$ are represented as functions of the corresponding pressures. The intersection point of these curves indicates that phasetransition occurs at a critical pressure nearly $P_{0}=8.810^{-2} \mathrm{fm}^{-4}$. At low pressure the local minimum of the Gibbs potential per particle corresponds to pure nuclear matter, while at pressures beyond $P_{0}$ the stable state corresponds to pure dibaryon matter. In order to construct the EOS we have drawn Fig. 3 with the pressure of Eq.(14), corresponding to $Q=0$ and $Q=0.5$ as functions of baryon density. The value $P_{0}$ is reached at baryon densities of $\rho_{I}=2.55 \rho_{0}$ in nuclear matter and $\rho_{I I}=3.18 \rho_{0}$ in the dibaryon condensate. Therefore it is a first-order phase-transition with a discontinuity jumping $0.63 \rho_{0}$ in the baryon density. The horizontal segment $P=P_{0}$ between $\rho_{I}$ and $\rho_{I I}$ represents two-phase coexistence. The effect of the phase-transition is to reduce the compressibility at high densities. For example, the quotient of the thermodinamical compressibility evaluated in the dibaryon condensate to its value in the nuclear matter gives at $\rho_{B}=3.5 \rho_{0}$ the value 0.706. This fact could be favorable for the collapse of very massive stars [20]. In Table I a comparison of the quotient of thermodinamical compressibility at $Q=0$. and $Q=0.5$ for several values of $M_{D}$ at the transition point is shown.

In addition, we have studied how much is modified the phase-transition point as 
the in-vacuum dibaryon mass $M_{D}$ is changed. It has been found that the density $\rho_{I}$ is an increasing function of the mass $M_{D}$, as it is shown in Table I. We must take into account that dibaryons have not been observed at densities around the normal saturation density $\rho_{0}$ and at sufficiently high densities our model can not describe the quark-gluon plasma phase-transition. Therefore we limit the search for the nuclear-dibaryon phasetransition at densities ranged from $1.5 \rho_{0}$ to $4 \rho_{0}$. This requirement constrains the variation of dibaryon mass to $1940 \mathrm{MeV}<M_{D}<2000 \mathrm{MeV}$.

A comparison with the hadronic field theoretical model of reference [9] shows that in the Hartree approximation a nuclear-dibaryon heterophase appears rather than a pure condensate. The Bose-condensate for $d^{\prime}$ dibaryon appears at 3 times the saturation density of nuclear matter. Independent calculations using the quantum field theory of hadrons and dibaryons have been developed in [21]. In this framework the transition density for nuclear and neutron matter is $\rho_{D} / \rho_{0}=2.87$ and $\rho_{D} / \rho_{0}=2.16$ respectively [22], similar results are found in reference [23]: $\rho_{D} / \rho_{0}=2.69$ and $\rho_{D} / \rho_{0}=2.57$ for nuclear and neutron matter, respectively. Therefore our prediction of $\rho_{D} / \rho_{0}=2.55$ is consistent with these values.

In this work we have studied the properties of a system composed of symmetric infinite nuclear matter with dibaryons. This has been performed in a theoretical framework which takes into account the finite size as well as the quark structure of nucleons and dibaryons. The variation of particle properties with baryon density have also been considered. The nucleon mass shows a monotonic decreasing behavior for all the densities and dibaryon abundances $Q$ studied here. At $Q=0$ nucleon swelling is observed at densities below the normal nuclear matter density. The bag radius increment is less than $2 \%$, in accordance with theoretical estimates based on y-scaling interpretation of quasi-elastic electron scattering [19]. A nuclear-dibaryon matter phase-transition is found at zero temperature. The dibaryon condensate predicted in this work could be an intermediate state before the transformation to quark matter. If a dibaryon condensation takes place for baryon densities in the range $1.5<\rho_{B} / \rho_{0}<4$, then values $1940 \mathrm{MeV}<M_{D}<2000 \mathrm{MeV}$ are predicted for non-strange dibaryon masses. As a consequence of phase transition the compressibility of the system is considerably reduced at high densities, as it should be expected from astrophysical scenarios.

Extensions of the present work in order to describe strange dibaryons can be inmediately performed by inclusion of the color electric and color magnetic interactions in the MIT bag model, also multiquark bags describing some exotic states of multibaryons could be treated in this framework.

This work has been supported in part by the grant PMT-PICT0079 of ANPCYT, Argentina. M.S. has been supported in part by The Nuclear High Energy Physics Research Center of Hampton University, USA, and by a grant of the Fundación Antorchas, Argentina. We have been beneficted from stimulating discussions with J.E. Horvath (IAG- 
USP, Brazil). M.S. is greatly indebted to J.L. Goity (Jefferson Lab-Hampton University) for kind hospitality at Jefferson Lab.

\section{References}

[1] A. Chodos, R. L. Jaffe, K. Johnson and C. B. Thorn, Phys. Rev. D 10 (1974) 2599.

[2] E. Fahri and R.L. Jaffe, Phys. Rev. D 30 (1984) 2379; E.P. Gilson and R.L. Jaffe, Phys. Rev. Lett. 71 (1993) 332.

[3] R. L. Jaffe, Phys. Rev. Lett. 38 (1977) 195.

[4] S.V. Bashinsky, R.L. Jaffe, Nucl. Phys. A 625 (1997) 167; T. Goldman, K. Maltman, G.J. Stephenson, Jr., Jia-Lun Ping, Fang Wang; Mod. Phys. Lett. A 13 (1997) 59; S.B. Gerasimov, nucl-th/9712064; T. Sakai, J. Mori, A.J. Buchmann, K.Shimizu, K. Yazaki, Nucl. Phys. A 625 (1997) 192; Stathes D. Paganis, Takeshi Udagawa, G.W. Hoffmann, R.L. Ray, Phys. Rev. C 56 (1997) 570.

[5] G.L. Thomas, N.N. Scoccola and A. Wirzba, Nucl. Phys. A 575 (1994) 623, and references therein.

[6] M. Schvellinger and N.N. Scoccola, hep-ph/9801347 (Phys. Lett. B, in press).

[7] W. Brodowski et al., Z. Phys. C 355 (1996) 5; H. Clement, M. Schepkin, G. J. Wagner and O. Zaboronsky, Phys. Lett. B 337 (1994) 43; R. Bilger, H. A. Clement and M. G. Schepkin, Phys. Rev. Lett. 71 (1993) 42; R. Bilger et al., Phys. Lett. B 269 (1991) 247.

[8] A. Faessler, A. J. Buchmann, M. I. Krivoruchenko and B. V. Martemyanov, J. Phys. G 24 (1998) 791.

[9] A. Faessler, A. J. Buchmann, M. I. Krivoruchenko and B. V. Martemyanov, Phys. Lett. B 391 (1997) 255.

[10] P. A. M. Guichon, Phys. Lett. B 200 (1988) 235.

[11] K. Saito and A. W. Thomas, Phys. Lett. B 327 (1994) 9; K. Saito and A. W. Thomas, Phys. Rev. C 51 (1995) 2757.

[12] P.A.M. Guichon, K. Saito, E. Rodionov and A. W. Thomas, Nucl. Phys. A601 (1996) 349; K. Saito, K. Tsushima, A.W. Thomas, Nucl. Phys. A609 (1996) 339; P.G. Blunden, G.A. Miller, Phys. Rev C54 (1996) 359 
[13] K. Saito, A. Michels, A.W. Thomas, Phys. Rev C46 (1992) R2149; A.W. Thomas, K. Saito, A. Michels, Aust. J. Phys. 46 (1993) 3; K. Saito, A.W. Thomas, Nucl. Phys. A574 (1994) 659.

[14] K. Saito, K. Tsushima, A.W. Thomas, Phys. Lett. B 406 (1997) 287.

[15] X. Jin and B. K. Jennings, Phys. Lett. B 374 (1996) 13; Phys. Rev. C 54 (1996) 1427; Phys. Rev. C 55 (1997) 245.

[16] R. Aguirre and M. Schvellinger, Phys. Lett. B 400 (1997) 245.

[17] R. Aguirre and M. Schvellinger, University of La Plata Preprint, La Plata-Th-97/03, nucl-th/9704010.

[18] J. Zimanyi and S.A. Moszkowski; Phys. Rev. C 42 (1990) 1416.

[19] I. Sick; Phys. Rev. Lett. 45 (1980) 871.

[20] E. Baron, J. Cooperstein, and S. Kahana, Nucl. Phys. A440 (1985) 744; Phys. Rev. Lett. 55 (1985) 535; T. L. Ainsworth, E. Baron, G. E. Brown, J. Cooperstein and M. Prakash, Nucl. Phys. A 464 (1987) 740.

[21] N.K. Glendenning, J. Schaffner-Bielich; astro-ph/9803232; A. Faessler, A.J. Buchmann and M.I. Krivoruchenko, Phys. Rev. C 56 (1997) 1576; A. Faessler, A.J. Buchmann, M.I. Krivoruchenko and B.V. Martemyanov, nucl-th/9706079.

[22] J. E. Horvath, private communication.

[23] S. Kagiyama, A. Nakamura and T. Omodaka, Z. Phys. C 56 (1992) 557. 


\begin{tabular}{cccc}
$M_{D}[\mathrm{GeV}]$ & $\rho_{I}\left[\mathrm{fm}^{-3}\right]$ & $\rho_{I I}\left[\mathrm{fm}^{-3}\right]$ & $\kappa_{I I} / \kappa_{I}$ \\
\hline \hline 1.94 & 0.228 & 0.335 & 1.30 \\
1.95 & 0.283 & 0.380 & 1.09 \\
1.96 & 0.335 & 0.429 & 1.01 \\
1.97 & 0.383 & 0.477 & 0.98 \\
1.98 & 0.427 & 0.522 & 0.96 \\
1.99 & 0.470 & 0.566 & 0.94 \\
2.00 & 0.505 & 0.603 & 0.93 \\
\hline
\end{tabular}

Table 1: The baryon density $\rho$ and the quotient of thermodinamical compressibility $\kappa=\partial P / \partial \rho_{B}$ evaluated at the transition point as functions of the in-vacuum dibaryon mass $M_{D}$. The label I (II) corresponds to pure nuclear (dibaryon) matter state.

Figure 1: $B$ (dashed line) and the nucleon bag radius, $R_{1}$, for the values $Q=0,0.25$, $0.33,0.47$ and 0.5 (solid lines) as functions of the baryon density, $\rho_{B}$. The arrow indicates the increasing $\mathrm{Q}$ values. $R_{N}$ is the nucleon radius at zero baryon density.

Figure 2: The dibaryon chemical potential (dashed line) evaluated at $Q=0.5$ and two times the nucleon chemical potential (solid line) at $Q=0$ plotted as functions of the total pressure of the system. The phase-transition for in-vacuum dibaryon mass $M_{D}=1970 \mathrm{MeV}$ occurs at a baryon density of $\rho_{I}=2.55 \mathrm{fm}^{-3}$ in the nuclear matter phase, corresponding to a baryon density of $\rho_{I I}=3.17 \mathrm{fm}^{-3}$ in the pure dibaryon phase.

Figure 3: Pressure as a function of baryon density for the dibaryon abundances $Q=0$, $0.25,0.33,0.47$ and 0.5 (dashed lines). The arrow shows the increasing Q values. Solid line represents the physical states of the system, corresponding to nuclear matter $(Q=0)$ below the relative density $\rho_{B} / \rho_{0}=2.55$ and to pure dibaryon matter $(Q=0.5)$ above the value $\rho_{B} / \rho_{0}=3.17$. The horizontal segment between these densities represents a coexistence region between the two phases. 


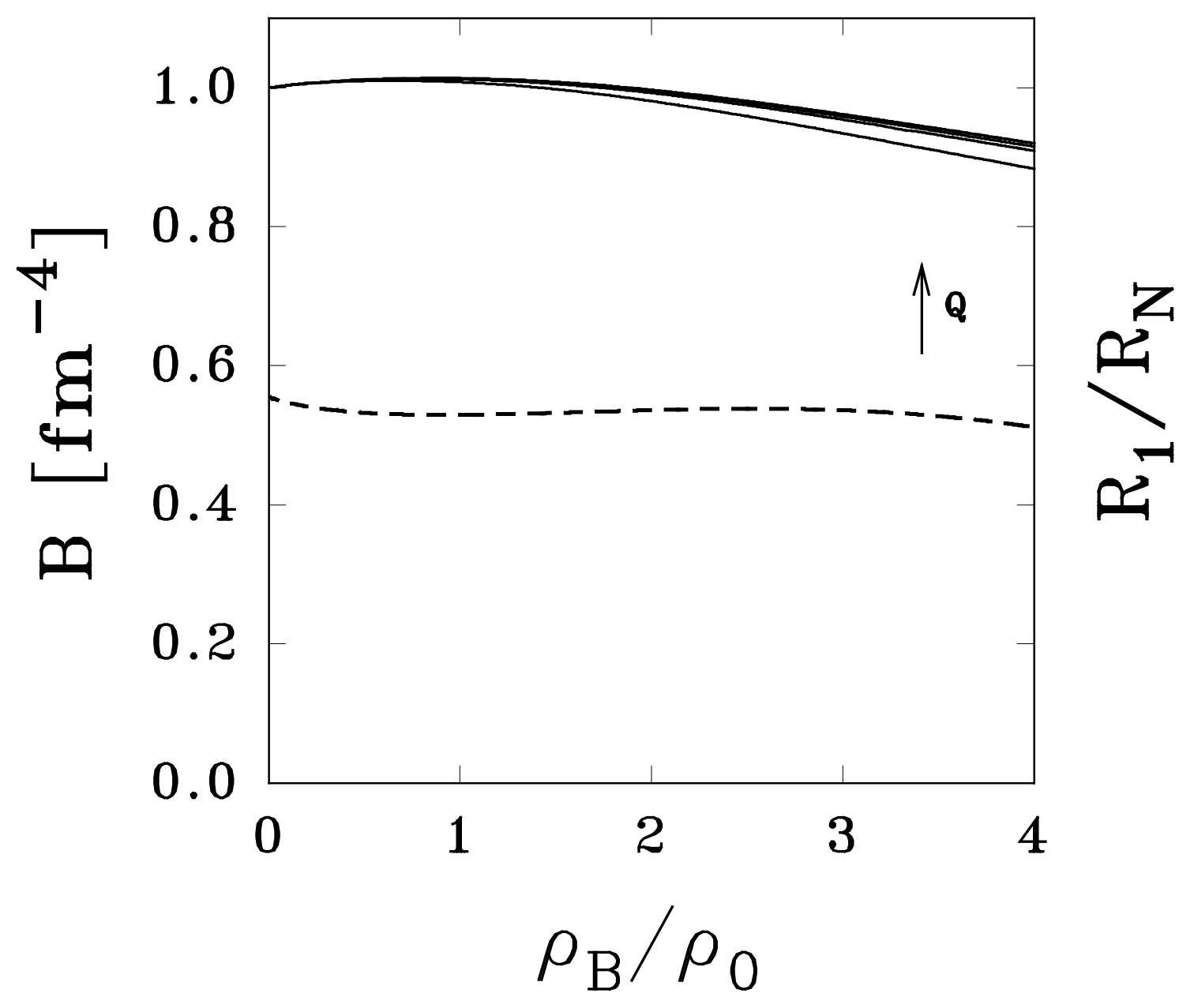




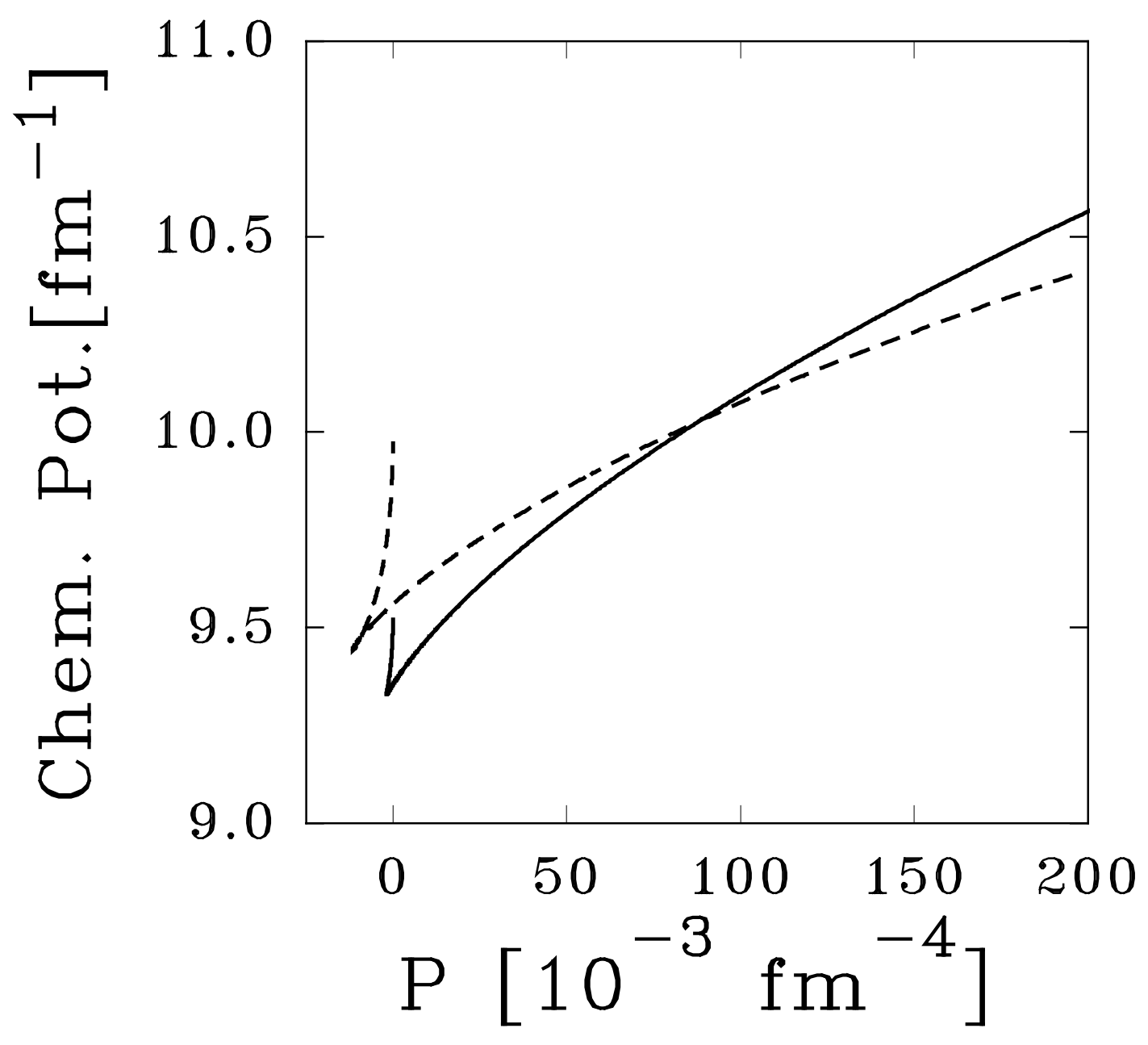




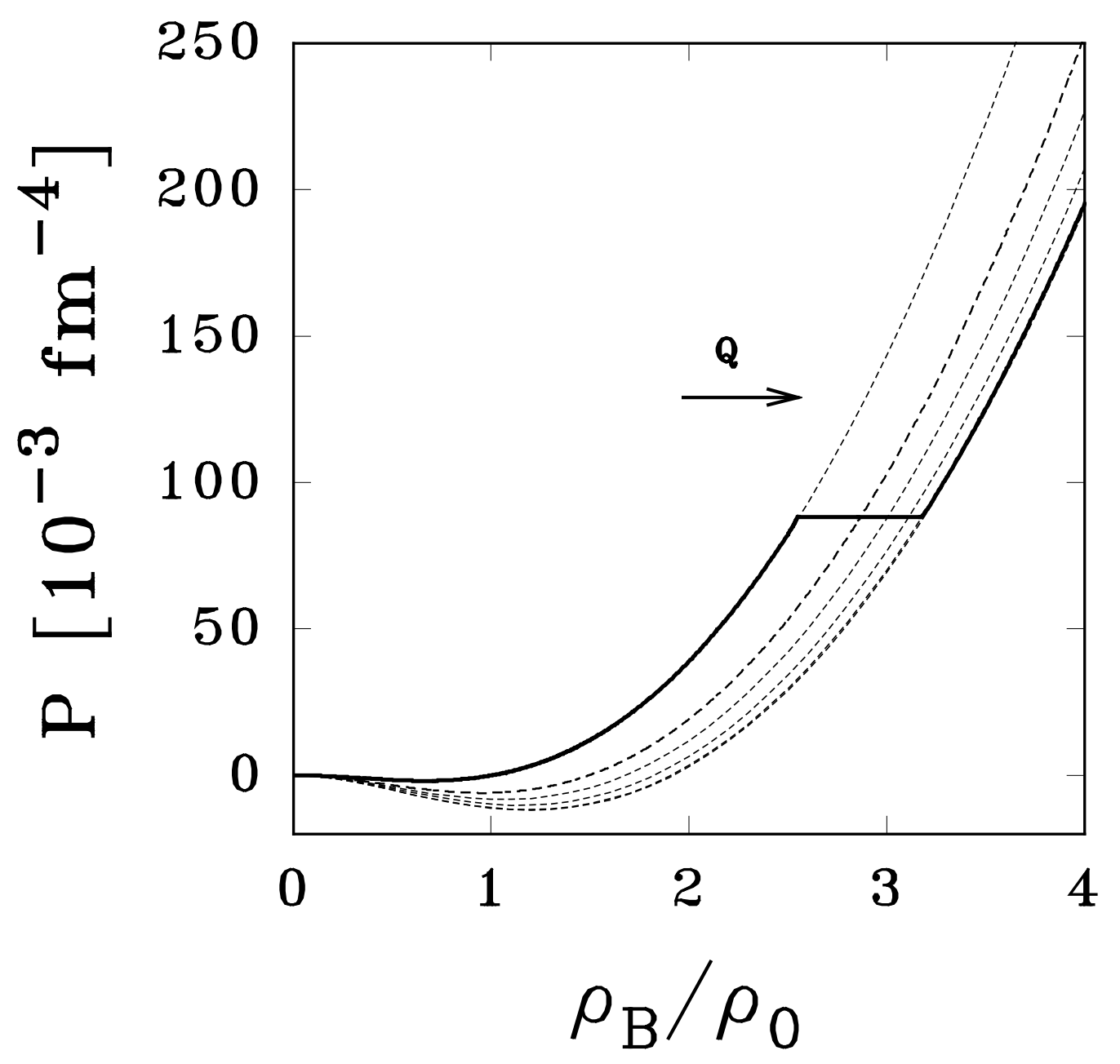

